\title{
Análise de níveis desenvolvimentais nas habilidades de controle de objetos em pré-escolares
}

http://dx.doi.org/10.11606/1807-5509201800010109

\author{
Maria Teresa CATTUZZO* \\ Ilana Santos de OLIVEIRA** \\ Dayana da Silva OLIVEIRA ${ }^{* * * *}$ \\ Natália Barros BELTRÃO ${ }^{* * * * *}$ \\ Teresinha de Jesus Sousa LIMA* \\ Anderson Henry Pereira FEITOZA*
}

${ }^{*}$ Escola Superior de Educação Física,

Universidade de

Pernambuco, Recife,

PE, Brasil.

** Universidade

Federal de

Pernambuco, Recife,

PE, Brasil.

*** Universidade

Estácio de Sá Recife

Recife, PE, Brasil.

**** Universidade

Federal Rural de

Pernambuco, Recife,

PE, Brasil.

\section{Resumo}

0 contato intencional e controlado com objetos é a meta das habilidades de controle de objetos e espera-se que as mudanças em tais habilidades apareçam em uma sequência progressiva de níveis desenvolvimentais, com o aumento da idade cronológica. 0 sexo é um fator a ser melhor estudado neste contexto. Este estudo investigou os níveis desenvolvimentais (inicial, intermediário e avançado) em habilidades de controle de objetos em pré-escolares, segundo idade e sexo. A amostra foi composta por 342 crianças, de 3, 4 e 5 anos (187 meninos). 0 TGMD-2 foi usado para avaliar o desempenho nas habilidades rebater, quicar, receber, chutar, arremessar e rolar. Diferenças entre grupos e sexos foram analisadas com testes não paramétricos; o teste Quiquadrado foi usado para identificar o percentual de crianças nos niveis desenvolvimentais das habilidades, em cada idade. As crianças de 5 anos foram superiores às de 3 anos no quicar, receber e escore total de controle de objetos; nas comparações entre crianças de 3 e 4, e 4 e 5 anos, a diferença ocorreu a favor das crianças mais velhas apenas no quicar e escore total. Os meninos apresentaram desempenho superior em cinco das seis habilidades e no escore total. Houve associação entre os mais avançados níveis desenvolvimentais e 0 sexo masculino para o escore total (aos 3 e 5 anos), e para as habilidades rebater e rolar (4 anos) e arremessar (aos 4 e 5 anos). Em geral, o desempenho dos pré-escolares nas habilidades de controle de objetos melhorou com o avanço da idade, evidenciando que o desenvolvimento motor é cumulativo e progressivo.

Palavras-chave: Desenvolvimento Infantil; Desempenho Psicomotor; Criança; Destreza Motora.

\section{Introdução}

A competência na realização de habilidades motoras durante a infância parece condicionar o curso do desenvolvimento humano saudável. Isso porque inúmeras vantagens têm sido destacadas em decorrência de uma boa competência motora, tais como uma maior percepção de competência atlética $^{1,2}$, maior autonomia no ambiente, prevenção da obesidade na infância, além de influências positivas na saúde geral da criança ${ }^{3}$.
Um indivíduo que tem competência motora sabe realizar as ações motoras, motiva-se para realizá-las, mostra confiança no seu desempenho e continua movimentando-se por toda a vida ${ }^{4}$. Resultados de pesquisas longitudinais têm sugerido que, em especial, o domínio nas habilidades de controle de objetos na infância pode influenciar positivamente o futuro engajamento em atividade física e a aptidão física na adolescência ${ }^{5}$. Assim, o desenvolvimento 
motor além de ser progressivo e cumulativo ${ }^{6}$, parece manter uma relaçáo dinâmica e sinérgica com outras variáveis comportamentais ${ }^{7,8}$, concorrendo para um estilo de vida ativo e saudável.

O desenvolvimento motor ativo e saudável ao longo da vida repousa sobre um pressuposto central, que é a ideia de mudanças em direção à maestria em habilidades motoras ${ }^{7}$. As habilidades motoras de locomoção, de controle de objetos e de equilíbrio são fundamentais para a participação na maioria das atividades físicas, porque elas representam os requisitos comportamentais necessários para tal participação?. Numa perspectiva sistêmica ${ }^{10,11}$ o comportamento humano é um fenômeno dinâmico, complexo e aberto e, esta visão também pode ser aplicada à compreensáo da maestria em habilidades motoras. Assim, a competência motora é um atributo adaptável gerado por meio das interaçóes entre restrições de fatores biológicos, psicológicos, instrucionais e ambientais que mudam ao longo do tempo, sendo essas mudanças relacionadas com a idade cronológica, mas não determinadas por ela ${ }^{8}$.

Nas perspectivas sistêmica ${ }^{10,11}$ e desenvolvimental ${ }^{12-14}$ está a noção de que a mudança se dá por meio de uma progressáo de comportamentos qualitativamente diferentes (novos); a descrição desses comportamentos em uma habilidade específica, ao longo do tempo de vida, forma a sequência de níveis desenvolvimentais intratarefa ${ }^{12}$. Segundo Haywood, Roberton e GETCHELL ${ }^{12}$, a descrição dos níveis desenvolvimentais pode focar o corpo total, ao descrever os níveis progressivos da configuração total do corpo, ou pode focar os níveis dos componentes corporais (açōes separadas do braço, tronco, pernas). Ambas, no entanto, compartilham a noção de que, cada nível da sequência tem uma probabilidade de aparecer dependendo da idade do indivíduo, sendo que os níveis mais avançados de desempenho, teoricamente, tem maior probabilidade de aparecerem entre os indivíduos mais velhos.

A análise das mudanças nos níveis desenvolvimentais intratarefa é um meio de retratar o trajeto desenvolvimental da competência em habilidades motoras na infância. O "domínio" em uma habilidade representa o estado mais avançado, e alguns autores têm proposto que quando a criança apresenta o desempenho correto em todos os componentes corporais avaliados, ela tem esse domínio; um estado menos avançado seria o "domínio-aproximado", que é quando a criança falha em mostrar o desempenho correto em todos os componentes de uma habilidade motora ${ }^{9,15}$.
Renomadas estudiosas sobre o assunto, HaYwood, Roberton e GETCHELL ${ }^{12}$ chamam a atenção para o fato de que, embora o conhecimento sobre os níveis de desenvolvimento intratarefa tenham uma presumível relevância para a pedagogia e que os livros-textos descrevam vários deles, pouca pesquisa tem sido feita para investigar tais níveis desenvolvimentais, tanto em habilidades locomotoras quanto de controle de objetos.

O contato intencional e controlado com os objetos é a meta das habilidades motoras de controle de objetos. Esse controle envolve a estimativa da trajetória, distância, velocidade, precisão e massa do objeto em movimento, permitindo uma maior noção ao indivíduo acerca desses elementos ${ }^{16}$, e a execução de movimentos complexos de controle de objetos, os quais são utilizados em diversos esportes $^{5,16-19}$. A habilidade de arremessar sobre o ombro tem sido extensamente investigada na literatura ${ }^{12,20}$, assim como as habilidades de chutar e rebater ${ }^{21}$. Porém a mesma atençáo não tem sido dada para outras habilidades de controle de objetos, como o receber, quicar ou rolar uma bola.

Relativo à comparação entre sexos, pode-se esperar divergências no desempenho motor de meninos e meninas. Meninos parecem ter melhor desempenho motor, especialmente em habilidades de controle de objetos $^{19,22-25}$ e tal superioridade tem sido justificada devido ao maior incentivo e prática em atividades com bola, ofertada aos meninos. Entretanto, outras investigaçóes não confirmam esses achados, e evidenciam similaridade entre os sexos ${ }^{26-28}$. Enfim, não há respostas conclusivas a esse respeito, indicando que esta variável deve continuar sendo investigada.

Considerando o princípio da universalidade e da progressividade ${ }^{6}$, pode-se esperar que a maioria das crianças em qualquer parte do mundo apresentem melhores desempenhos motores à medida que ficam mais velhas. Assim, espera-se que os desempenhos classificados em níveis iniciais (imaturos/com falta de domínio ou maestria) sejam paulatinamente substituídos por níveis mais avançados (maduros/com domínio ou maestria). No entanto, na atualidade, o uso de tecnologia é, de fato, parte do cotidiano de primeira infância ${ }^{29}$, e tem contribuindo para um estilo de vida infantil cada vez mais sedentário ${ }^{30}$. Assim, parece plausível questionar se e como está ocorrendo a progressão do desenvolvimento motor infantil. Até o presente momento não se tem registro de estudos sobre o desenvolvimento da maestria em habilidades de controle de objetos em pré-escolares brasileiros. 
Diante do exposto, o presente estudo objetiva investigar os níveis desenvolvimentais em habilidades de controle de objetos de préescolares, em função da idade cronológica e sexo.

\section{Método}

Este é um estudo transversal, com enfoque quantitativo, de comparação entre grupos e correlacional $^{31}$. Este estudo está baseado em um levantamento epidemiológico de abrangência municipal, na cidade do Recife, intitulado ELOSPRÉ (Estudo Longitudinal de Observação da Saúde e Bem-estar da Criança em Idade Pré-escolar). O protocolo do estudo foi aprovado por um Comitê de Ética local (CAAE: 0096.0.097.000-10). Todos os participantes tiveram o Termo de Consentimento Livre e Esclarecido assinado pelos pais ou responsável.

A população foi composta por crianças de 3 a 5 anos matriculadas nas escolas da rede pública e privada de todas as seis Regióes Político-
Administrativas (RPAs) da cidade do Recife-PE. A seleção da amostra foi feita por conglomerado: inicialmente foram sorteadas as escolas, de modo a garantir representatividade das seis RPAs, e posteriormente, foram selecionadas as turmas as quais as crianças deveriam ser convidadas. Nesse levantamento, 543 crianças tiveram seus dados motores coletados. Para o presente estudo, a amostra foi composta a partir dos seguintes critérios de inclusão: (a) ter completado as duas tentativas de cada habilidade do subteste de controle de objetos. Assim, a amostra final deste estudo foi constituída por 348 crianças, sendo 190 meninos e 158 meninas (TABELA 1 ).

TABELA 1 - Quantitativo (n), média e desvio padrão da idade (meses) dos meninos e meninas de 3, 4 e 5 anos que participaram do subteste das habilidades de controle de objetos, Recife-PE.

\begin{tabular}{llcccccc}
\hline & \multicolumn{3}{l}{ 3 anos } & \multicolumn{3}{c}{ 4 anos } & \multicolumn{2}{c}{ 5 anos } \\
\hline & N & Média (DP) & N & Média (DP) & N & Média (DP) & N \\
\hline Menino & 40 & $42,6(4,8)$ & 66 & $55,0(3,9)$ & 81 & $65,6(3,0)$ & 187 \\
Menina & 24 & $41,0(3,8)$ & 62 & $55,7(3,0)$ & 69 & $66,0(3,3)$ & 155 \\
Total & 64 & $42,1(4,5)$ & 128 & $55,4(3,5)$ & 150 & $65,7(3,1)$ & 342 \\
\hline
\end{tabular}

DP - desvio padrão da idade em anos; $\mathrm{n}$ - número de sujeitos na amostra.
Para a verificação do desempenho nas habilidades de controle de objetos foi utilizada a segunda versão do Test of Gross Motor Development - TGMD-2 ${ }^{32}$, instrumento destinado a avaliar habilidades motoras que requerem a ação de grandes grupos musculares (locomotoras e de controle de objetos) em crianças de 3 a 10 anos de idade. Este teste apresentou índices satisfatórios de validade e consistência em crianças brasileiras $^{33}$. Na aplicação, cada criança era testada por dois avaliadores treinados. Um avaliador explicava e demonstrava a habilidade e, em seguida, a criança executava a mesma habilidade três vezes. A primeira execução realizada pela criança era um ensaio. Se o avaliador identificasse qualquer problema de entendimento por parte dela, ele realizava uma nova demonstração e, na sequência, as duas tentativas da criança eram filmadas.

As habilidades foram filmadas por câmera Digital Sony Cyber-Shot DSC-H20 (10.1 Megapixel) e posteriormente analisadas com reprodutor de vídeo
Media Player Classic (free download), em velocidade lenta. Os desempenhos eram pontuados segundo critérios da lista de checagem do teste: era atribuído um (1) ponto se o critério da lista de checagem fosse atendido, ou zero (0) caso contrário. A medida de desempenho foi o somatório dos pontos das duas tentativas (escore bruto). A pontuaçáo foi feita, de forma independente, por dois observadores treinados. A Concordância Entre Observadores (CEO) calculada de acordo com Thomas, Nelson e Silverman ${ }^{31}$ foi de $97 \%$. Nos casos de discordância, os dois avaliadores se reuniam e entravam em consenso.

Os níveis de desenvolvimento nas habilidades de controle de objetos foram calculados a partir dos escores brutos do teste (somando-se as duas tentativas), e divididos em tercis. Assim, definimos operacionalmente o desempenho dos pré-escolares em habilidades de controle de objetos como níveis de desenvolvimento da maestria/domínio dos elementos-chaves, na seguinte progressão: 
$1^{\circ} \cdot$ Tercil = 'inicial', que significa que a criança mostrou baixa maestria na realização dos elementoschaves das habilidades de controle de objetos, 20. Tercil = 'intermediário' que significa que a criança demonstrou maestria mediana na realização dos elementos-chaves das habilidades e $3^{\circ}$. Tercil = 'avançado' que significa que a criança mostrou domínio - maestria - na maioria dos elementoschaves das habilidades. Os escores brutos foram usados uma vez que os escores padróes do TGMD-2 estão baseados em curvas geradas apenas para a população norte-americana e também pela recomendaçáo de que os escores brutos do TGMD-2 servem bem a propósitos de pesquisa ${ }^{32}$.

No plano analítico, as variáveis antropométricas massa $(\mathrm{kg})$ e estatura $(\mathrm{cm})$ foram usadas para descrever as características da amostra. As variáveis independentes foram idade (3, 4 e 5 anos) e sexo. As variáveis dependentes foram os escores brutos obtidos em cada uma das seis habilidades de controle de objetos (rebater, quicar, receber, chutar, arremessar e rolar) e o escore total, calculado por meio da soma dos escores brutos das seis habilidades. Após a verificação da ausência de normalidade (teste de Kolmogorov-Smirnov), os dados de desempenho foram analisados por meio de estatística descritiva (mediana e intervalo interquartil). Para analisar a diferença entre sexos foi utilizado o teste U de Mann-Whitney; o tamanho do efeito foi calculado pelo $d$ de Cohen. Para analisar os níveis de desenvolvimento (inicial, intermediário e avançado) e identificar o percentual de indivíduos presentes em cada nível nas três idades avaliadas, foi utilizado o teste de qui-quadrado. $\mathrm{O}$ teste Exato de Fisher foi aplicado nos casos em que uma das células era menor ou igual a 5. Os dados foram organizados em planilhas do programa Microsoft Office Excel e analisados por meio dos pacotes estatísticos SPSS 10.0 e STATISTICA 5.1. Adotou-se um nível de significância de $(\mathrm{p} \leq 0,05)$.

\section{Resultados}

Relativo ao desempenho nas habilidades de controle de objetos (TABELA 2) houve diferença estatisticamente significante entre crianças de 3, 4 e 5 anos no escore total de controle de objetos ( $\mathrm{p}=$ 0,001 ). Ainda quanto ao escore total, crianças de 3 anos apresentaram desempenho inferior quando comparadas às de $4(\mathrm{p}=0,009)$ e 5 anos $(\mathrm{p}<0,001)$; não houve, entretanto, diferença estatisticamente significante entre as crianças de 4 e 5 anos de idade ( $\mathrm{p}=0,117)$.

Ao analisar cada uma das seis habilidades, as crianças de 4 anos mostraram desempenho superior às de 3 anos na habilidade receber $(\mathrm{p}=0,001)$. Crianças de 5 anos, além da habilidade receber $(\mathrm{p}<0,001)$, mostraram desempenho superior às de 3 anos na habilidade quicar $(p=0,010)$. Já na comparação entre 4 e 5 anos, houve diferença estatisticamente significante para a habilidade quicar $(\mathrm{p}=0,003)$.

$\mathrm{Na}$ análise comparativa entre sexos (TABELA 3), houve diferenças significantes no escore total das habilidades de controle de objetos, com desempenho superior dos meninos ( $\mathrm{p}<0,001) . \mathrm{Na}$ análise específica das habilidades, os meninos foram melhores no desempenho de todas as habilidades, exceto na habilidade receber, na qual não houve diferenças entre meninos e meninas $(\mathrm{p}=0,961)$.

Especificamente entre as crianças de 3 anos de idade, os meninos foram superiores no rebater $(p<0,001)$ e arremessar $(p=0,026)$. Entre as de 4 anos, houve superioridade dos meninos no rebater $(\mathrm{p}=0,039)$, chutar $(\mathrm{p}=0,023)$, arremessar $(\mathrm{p}=0,005)$ e rolar $(\mathrm{p}=0,006)$. $\mathrm{E}$ entre as crianças de 5 anos, os meninos mostraram desempenho superior nas habilidades quicar $(\mathrm{p}=0,024)$, chutar $(\mathrm{p}=0,008)$ e arremessar $(\mathrm{p}=0,003)$.

A FIGURA 1 informa a proporção (\%) de crianças de 3, 4 e 5 anos em cada nível desenvolvimental (inicial, intermediário e avançado), em todas as habilidades estudadas. Destaca-se a diminuiçáo da proporção de crianças exibindo nível desenvolvimental inicial em todas as habilidades, à medida que a idade avança; complementarmente, ocorreu o aumento da proporção de crianças exibindo o nível desenvolvimental mais avançado à medida que a idade aumenta. No entanto, a maior proporção de crianças está concentrada no nível desenvolvimental intermediário.

Numa análise mais detalhada, houve aumento na proporção de meninos no nível mais avançado, ou seja, exibindo maestria em suas açóes motoras, à medida que a idade cronológica avança nas habilidades: quicar (3 anos: 7,5\%; 4 anos: 7,6\%; 5 anos: $24,7 \%$ ), receber (3 anos: 7,5\%; 4 anos: 25,8\%; 5 anos: $28,4 \%$ ). O mesmo ocorre para a proporção de meninas que exibem um nível de desenvolvimento avançado nas habilidades rebater ( 3 anos: 16,6\%; 4 anos: 30,6\%; 5 anos: $49,3 \%$ ), receber ( 3 anos: $4,2 \%$; 4 anos: $21,0 \% ; 5$ 
anos: $29,0 \%$ ), arremessar ( 3 anos: $4,2 \%$; 4 anos: $8,1 \%$; 5 anos: $11,6 \%$ ) e no escore total (3 anos: $0,0 \%$; 4 anos: $8,1 \%$; 5 anos: $10,2 \%)$.

O qui-quadrado mostrou que houve associação entre os níveis de desenvolvimento e sexo para a habilidade arremessar $\left(\mathrm{x}^{2}=8,05 ; \mathrm{p}=0,01\right)$ aos 5 anos de idade. Nesta idade e habilidade, os meninos estão em maior proporção nos níveis intermediário e avançado quando comparados às meninas; no nível mais inferior da escala, ou seja, no primeiro tercil denominado inicial, as meninas aparecem em maior proporção que os meninos.

O teste Exato de Fisher identificou associação entre os níveis de desenvolvimento e sexo para o escore total (exato de Fisher $=6,49 ; \mathrm{p}=0,03$ ) aos 3 anos; quando comparadas aos meninos (inicial: $7,5 \%$; avançado: $10,0 \%$ ) , um maior porcentual das meninas (29,2\%) mostrou nível de desenvolvimento inicial, enquanto que nenhuma $(0,0 \%)$ atingiu o nível avançado, ou seja, domínio das habilidades. Aos 4 anos, foi encontrada associação entre os níveis de desenvolvimento e sexo para as habilidades rebater (exato de Fisher $=5,44 ; p=0,05$ ), arremessar (exato de Fisher $=9,69 ; \mathrm{p}=0,00)$ e rolar (exato de Fisher $=$ $7,90 ; p=0,01)$; novamente, as meninas obtiveram pior desempenho, considerando que no rebater o maior porcentual delas $(64,6 \%)$ mostraram nível de desenvolvimento intermediário e os meninos (50,0\%) mostraram nível de desenvolvimento avançado; 51,6\% das meninas mostraram nível de desenvolvimento inicial na habilidade arremessar e $54,8 \%$ na habilidade rolar, enquanto que os meninos já estavam no nível de desenvolvimento intermediário (36,4\% e $47,0 \%$, respectivamente). Relativo ao escore total de habilidades de controle de objetos houve associação entre os níveis de desenvolvimento e sexo para as crianças de 5 anos (exato de Fisher $=7,65 ; \mathrm{p}=0,02)$, nesse caso, um porcentual quase três vezes maior de meninos $(27,2 \%)$ mostrou o nível de desenvolvimento avançado nas seis habilidades de controle de objetos estudadas, quando comparados às meninas (10,2\%).

TABELA 2 - Mediana (Med), intervalo interquartil (IIQ) e tamanho do efeito ( $d$ de Cohen) da comparação entre crianças de 3 e 4 anos, 3 e 5 anos e 4 e 5 anos de idade do desempenho nas habilidades rebater, quicar, receber, chutar, arremessar, rolar e no escore total de controle de objetos (CO Total), Recife-PE.

\begin{tabular}{|c|c|c|c|c|c|c|c|c|c|}
\hline Habilidades & $\begin{array}{c}3 \text { anos } \\
\text { Med (IIQ) }\end{array}$ & $\begin{array}{c}4 \text { anos Med } \\
\text { (IIQ) }\end{array}$ & d & $\begin{array}{c}3 \text { anos } \\
\text { Med (IIQ) } \\
\end{array}$ & $\begin{array}{c}5 \text { anos } \\
\text { Med (IIQ) } \\
\end{array}$ & d & $\begin{array}{c}4 \text { anos } \\
\text { Med (IIQ) }\end{array}$ & $\begin{array}{c}5 \text { anos } \\
\text { Med (IIQ) } \\
\end{array}$ & d \\
\hline & & & 0,15 & & & 0,26 & & & \\
\hline Quic & & & & & & 26 & & & \\
\hline Receber & $2,0(2,0)$ & $3,0(3$ & 0,72 & $2,0(2$ & $4,0($ & 0,89 & & 4,0 & 0,17 \\
\hline Chutar & $6,0(2$ & & 005 & 6,0 & & 0,00 & & & 0,05 \\
\hline Arreme & $3,0(3,0)$ & $3,0(3,0)$ & 0,24 & $3,0(3,0)$ & $3,0(4,0)$ & 0,22 & $3,0(3,0)$ & $3,0(4,0)$ & 0,00 \\
\hline & & & 0,14 & & & 0,19 & & & 0,05 \\
\hline CO Total & $24,0(11,0)$ & $25,0(8,0)^{*}$ & 0,35 & $24,0(11,0)$ & $28,0(8,7)^{*}$ & 0,50 & $25,0(8,0)$ & $28,0(8,7)$ & 0,19 \\
\hline
\end{tabular}

* Diferença estatisticamente significante intergrupos $(p \leq 0,01)$.

TABELA 3 - Mediana (Med), intervalo interquartil (IIQ), nível de significância e tamanho do efeito ( $d$ de Cohen) do desempenho nas habilidades rebater, quicar, receber, chutar, arremessar, rolar e no escore total de controle de objetos em meninos e meninas de 3, 4 e 5 anos de idade, Recife-PE.

\begin{tabular}{|c|c|c|c|c|c|c|c|c|c|}
\hline \multirow[b]{2}{*}{ Habilidades } & \multicolumn{2}{|c|}{3 anos } & & \multicolumn{3}{|c|}{4 anos } & \multicolumn{2}{|c|}{5 anos } & \multirow[b]{2}{*}{ d } \\
\hline & $\begin{array}{c}\text { Meninos } \\
\text { Med (IIQ) } \\
\end{array}$ & $\begin{array}{c}\text { Meninas } \\
\text { Med (IIQ) }\end{array}$ & & $\begin{array}{c}\text { Meninos } \\
\text { Med (IIQ) }\end{array}$ & $\begin{array}{c}\text { Meninas } \\
\text { Med (IIQ) }\end{array}$ & d & $\begin{array}{c}\text { Meninos } \\
\text { Med (IIQ) }\end{array}$ & $\begin{array}{c}\text { Meninas } \\
\text { Med (IIQ) }\end{array}$ & \\
\hline Rebater & $7,2(2,1)^{*}$ & $5,5(2,5)$ & 0,77 & $7,0(3,0)^{*}$ & $7,0(2,0)$ & 0,34 & $8,0(3,0)$ & $7,0(2,0)$ & 0,22 \\
\hline Quicar & $3,0(3$ & $2,0(4$ & 0,05 & 2,0 & 2,0 & 0,12 & 4,0 & 3,0 & 0,37 \\
\hline Receber & $2,0(2,0)$ & $2,0(3$, & 0,15 & $4,0(3,0)$ & $3,0($ & 0,00 & $4,0(2,0)$ & 4,0 & 0,00 \\
\hline Chutar & $6,0($ & 5,0 & 0,36 & 7,0 & & 0,35 & 7,0 & 6,0 & 0,27 \\
\hline Arremessar & $3,0(2,2)^{*}$ & $1,0(3,0)$ & 0,80 & $4,0(4,0)^{*}$ & $3,0(2,0)$ & 0,58 & $4,0(4,0)^{*}$ & $2,0(4,0)$ & 0,57 \\
\hline Rolar & $2,0(3,0)$ & $4,0(5,5)$ & 0,26 & $4,0(3,0)^{*}$ & $2,0(2,0)$ & 0,44 & $3,0(3,0)$ & $3,0(3,0)$ & 0,05 \\
\hline Total & $24,0(9,5)^{*}$ & $22,0(13,5)$ & 0,56 & $28,0(7,0)^{*}$ & $23,0(6,0)$ & 0,63 & $29,0(7,0) *$ & $25,0(9,5)$ & 0,51 \\
\hline
\end{tabular}

* Diferença estatisticamente significante intragrupos $(p \leq 0,05)$. 


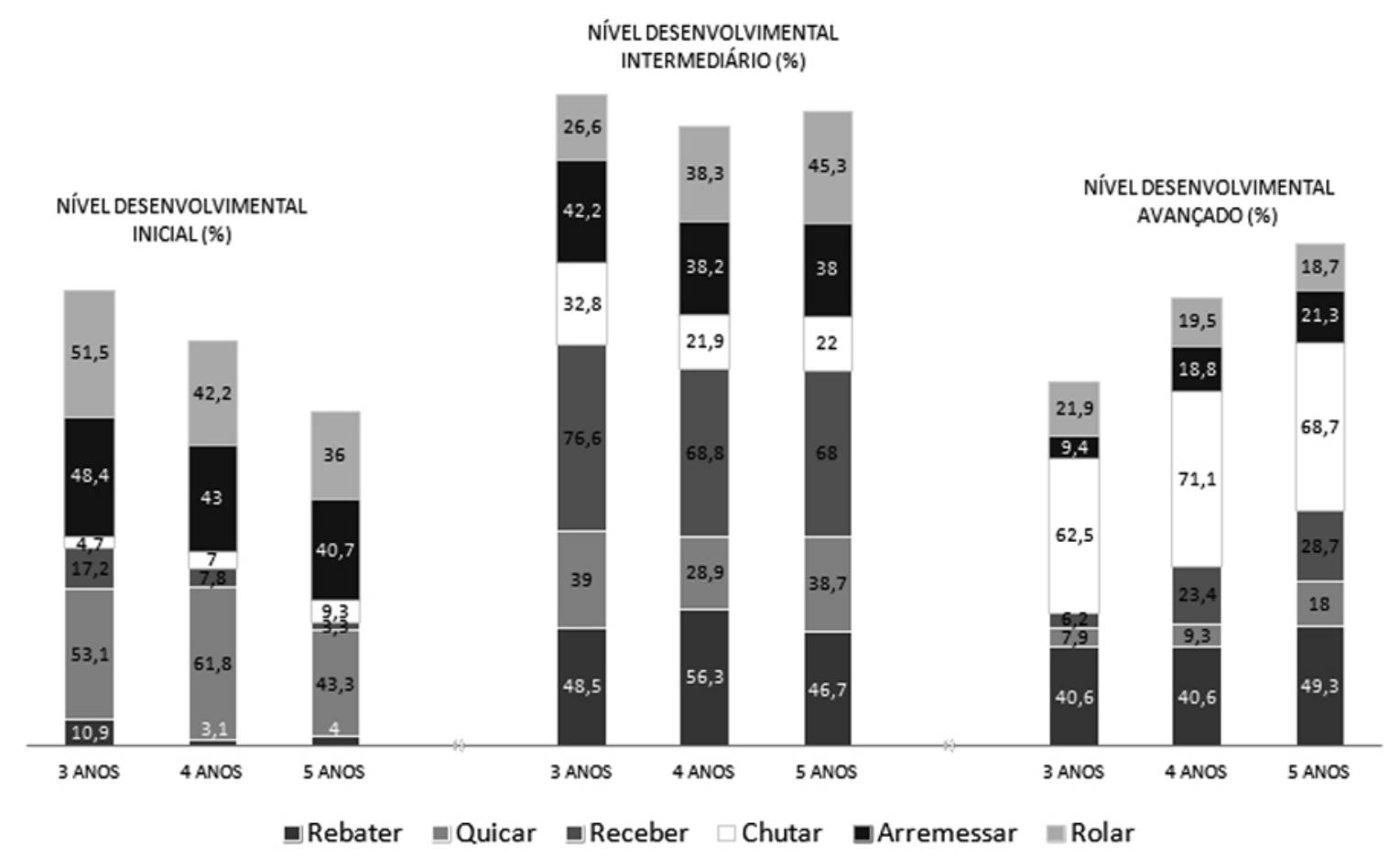

FIGURA 1 - Proporção (\%) de crianças em cada nível desenvolvimental (inicial, intermediário e avançado) aos 3, 4 e 5 anos. Recife. PE

\section{Discussão}

Este é um estudo transversal pré-longitudinal que teve como objetivo investigar níveis desenvolvimentais em habilidades de controle de objetos, em meninos e meninas pré-escolares de 3, 4 e 5 anos. Numa perspectiva desenvolvimental, este tipo de estudo tem o valor de permitir que o investigador faça a inferência de que as mudanças ocorreram no tempo a partir da diferença entre as idades dos grupos ${ }^{12}$.

$\mathrm{Na}$ comparação entre idades, alguns resultados apontaram que os desempenhos em habilidades de controle de objetos das crianças mais velhas foram superiores às mais novas, concordando com diversos estudos que evidenciaram a melhora no desempenho motor associada ao avanço da idade cronológica ${ }^{23,34-37}$. Pansera, De Paula e VALENTINI ${ }^{34}$, que analisaram crianças de 4 a 7 anos, PAIM $^{37}$, com crianças de 5 e 6 anos, e Afonso et al. ${ }^{23}$, que investigaram crianças de 3 a 10 anos de idade, identificaram uma melhoria ao longo da idade nas habilidades de quicar e rolar. O presente estudo corrobora esses resultados no que diz respeito à habilidade quicar, quando comparadas as crianças de 3 e 5 anos e 4 e 5 anos.
Ao considerar que crianças mais velhas têm vantagens em termos maturacionais e de quantidade de experiência prática nas diversas habilidades, um importante aspecto a ser considerado na melhora do comportamento motor com o avanço da idade é o melhor processamento da informaçáo relativo às instruçóes (verbal e demonstração) ofertadas nos testes. Segundo CowAN ${ }^{38}$, o desenvolvimento da memória de trabalho, que permite o armazenamento temporário de informação com capacidade limitada, ocorre, sobretudo, porque crianças tornam-se mais eficientes em focalizar a atenção na informação relevante e em inibirem aspectos irrelevantes, que muitas vezes são ativados automaticamente. Assim, as crianças mais velhas, tem certa vantagem, comparadas às mais novas, para lidarem com a informação verbal e visual oferecida pelos instrutores. Ratificando essa proposição, no presente estudo as crianças de 5 anos mostraram desempenhos superiores.

Uma possível justificativa para a similaridade no desempenho em algumas habilidades das crianças de diferentes faixas etárias, segundo VALENTINI ${ }^{39}$, é falta de oportunidades de práticas em que se manipulem objetos, como também, a carência de instrução 
específica nessas habilidades no decorrer dos anos. Ou seja, as condiçóes de prática são restrições que condicionam o avanço no domínio das habilidades motoras fundamentais. Fundamentando-se numa perspectiva de sistemas dinâmicos e complexos, SMITH e THELEN ${ }^{40}$ enfatizam que as experiências cotidianas da criança criam mudanças desenvolvimentais, tanto aquelas consideradas universais (em que toda criança deve exibir tais mudanças), como, especialmente, os trajetos individuais. Nessa perspectiva, o real domínio das habilidades motoras fundamentais depende de vários fatores ambientais, como oportunidades para a prática, encorajamento e ensino em ambiente propício ao aprendizado ${ }^{16,41-43}$.

Quanto ao sexo, diferenças significativas foram observadas nas habilidades rebater, quicar, chutar, arremessar, rolar e no escore total das habilidades de controle de objetos, sempre com desempenho superior dos meninos. Esse resultado corrobora os achados de outros estudos sobre habilidades de controle de objetos com crianças na idade préescolar ( 3 até 5 anos de idade) $)^{19,22,30}$. Os resultados do estudo de Hardy et al. ${ }^{19}$ com crianças de 2-6 anos de idade, mostraram exatamente os mesmos resultados que os nossos, pois os meninos tiveram o escore em habilidades de controle de objetos mais elevado para cada habilidade do TGMD-2, exceto receber. Em um estudo recente com crianças pré-escolares chinesas, YANG, LIN e TsAI ${ }^{30}$ encontraram que nas habilidades de controle de objetos os meninos foram superiores nas habilidades de rebater, chutar e arremessar.

Um conjunto de fatores ambientais, como os modelos culturais, os quais promovem e motivam de forma distinta os hábitos de prática motora, lúdica e desportiva entre os sexos, parece ser um fator determinante para essas diferenças entre meninos e meninas ${ }^{16,44}$. Segundo Carvalhal e VASCONCElos-Raposo ${ }^{24}$, quando se observam crianças em situação de atividade livre, os meninos apresentam superioridade em frequência e tempo de utilização de movimentos manipulativos e maiores oportunidades de prática e incentivos sociais parecem ser oferecidas a eles.

No estudo de XAVIER ${ }^{45}$, que investigou crianças de 6 e 7 anos, foi encontrado que as meninas obtiveram um maior índice de desempenho motor nas habilidades de controle de objetos. No entanto, o pequeno tamanho amostral do estudo (40 crianças) pode ter causado um viés importante. Além disso, a própria autora sugere que as condiçóes econômicas, os aspectos culturais e até mesmo as condições climáticas, possam ter influenciado nos resultados. Similaridade entre os sexos no desempenho das habilidades de controle de objetos também foram apontadas ${ }^{26-28}$, mas, o reduzido tamanho amostral desses estudos pode ser apontado como uma possível limitação.

De forma mais específica, o presente estudo identificou superioridade dos meninos no desempenho da habilidade chutar nas idades mais avançadas ( 4 e 5 anos). Essa habilidade, que está ligada a açôes típicas do futebol, esporte bastante difundido no Brasil, é praticada com maior frequência por meninos do que por meninas desde muito cedo na infância ${ }^{24}$. Além da questão maturacional, a questão cultural, uma restrição ambiental ${ }^{16}$, mais uma vez, parece explicar a diferença entre meninos e meninas, indicando que o aspecto cultural deve ser um forte componente na formação do padrão motor infantil.

Por outro lado, corroborando resultados de dois outros estudos ${ }^{19,30}$ na habilidade receber não houve diferença entre meninos e meninas em quaisquer das idades estudadas (3, 4 e 5 anos), poder-se-ia sugerir que tal habilidade tem poucas demandas perceptomotora que discriminem os sujeitos. Entretanto, a partir da análise dos níveis de desempenhos, foi possível observar que a maior proporção das crianças de 3, 4 e 5 anos apresentou o nível intermediário para a habilidade receber. Estudo com habilidades locomotoras de pré-escolares ${ }^{46}$ usando a matriz teórica proposta pelo modelo das restriçóes ${ }^{47}$ sugeriu que, além do estado maturacional (relacionado à idade) e sexo (ambas restriçóes do indivíduo), o alcance de um nível desenvolvimental avançado em uma habilidade motora está sujeito a outros fatores, como, por exemplo, a oportunidade de prática deliberada (restrições do ambiente) e dos requerimentos perceptuais e efetores específicos de uma dada habilidade (restriçóes da tarefa).

A análise dos níveis de desenvolvimento mostrou que há uma diminuição da proporção de crianças no nível inicial à medida que a idade avança, corroborando a hipótese teórica do presente estudo. Também, parece bastante plausível que a maioria das crianças tenha apresentado o nível intermediário na maioria das habilidades, uma vez que são crianças muito jovens. O nível avançado, que reflete domínio/maestria na habilidade, está aumentando progressivamente à medida que a idade avança, mas ainda não é o de maior proporção, pois a probabilidade maior é que isso ocorra mais tarde ${ }^{12}$.

$\mathrm{O}$ resultado de que a maior proporção das crianças encontra-se num nível de desenvolvimento 
intermediário para a maioria das habilidades de controle de objetos, também pode ser justificado pelo fato de que, de forma geral, crianças préescolares ainda náo são capazes de cumprir e ultrapassar uma ampla carga de tarefas cognitivas e motoras ${ }^{16}$. O estudo de Afonso et al. ${ }^{23}$ utilizou a classificação do TGMD-2 em categorias de desempenho motor em crianças de 3 a 10 anos, e identificou que o maior aglomerado de crianças da amostra estudada encontrava-se nas categorias "média" e "abaixo da média" em habilidades de controle de objetos, o que confirma a falta de domínio motor das crianças mais novas.

No presente estudo, a habilidade chutar foi aquela na qual a maior proporção de crianças de 3, 4 e 5 anos de idade atingiu o nível de desenvolvimento mais avançado. Uma possível explicação para esse fato é que, entre as habilidades avaliadas no teste TGMD-2, o chutar é uma daquelas nas quais os critérios parecem ser muito fáceis de serem cumpridos pelas crianças, ocorrendo o chamado efeito-teto. Crianças de cinco anos também atingiram o nível avançado para a habilidade rebater, no entanto, as crianças mais novas (3 e 4 anos) apresentaram nível intermediário.

Como dito anteriormente, o futebol, caracterizado principalmente pela habilidade de chutar, é um esporte bastante difundido no Brasil, o que pode ter estimulado a prática dessa habilidade, especialmente entre os meninos. O mesmo estímulo ambiental não acontece para a habilidade de rebater, além disso, ela é uma das habilidades motoras fundamentais de mais difícil julgamento perceptivo ${ }^{21}$, mesmo assim, uma porcentagem expressiva dos meninos, nas três idades analisadas, atingiu o nível de desenvolvimento avançado.

Consideramos que uma limitação deste estudo foi a ausência do rastreamento de quais crianças estavam envolvidas com práticas motoras sistemáticas, o tipo de instrução e nível de aptidão física, uma vez que já há indícios que pré-escolares que tiveram instrução e prática de habilidades motoras mostraram os melhores desempenhos em testes de habilidade motoras fundamentais ${ }^{48-50}$, e também a associação inequívoca entre a competência motora e a aptidão física ${ }^{51}$. Assim, a condição de interação indivíduocontexto e a multicausalidade desenvolvimental ${ }^{52}$ devem ser mais bem exploradas em estudos posteriores sobre a competência motora infantil.

De acordo com os achados deste estudo as crianças mais velhas sempre mostraram melhores desempenhos, mas não em todas as habilidades investigadas. Uma vez que ocorreram mudanças desenvolvimentais progressivas no comportamento motor dos pré-escolares, reforça-se a noção teórica que os níveis mais avançados de desenvolvimento devem aparecer entre os indivíduos mais velhos ${ }^{12}$. Relativo às diferenças entre sexos, os meninos foram superiores na maioria das habilidades analisadas e no escore total, assim como mostraram o mais avançado nível desenvolvimental nas habilidades rebater e rolar (quatro anos), no arremessar (aos quatro e cinco anos) e no escore total (aos três e cinco anos). Enfim, conclui-se que nas habilidades de controle de objetos investigadas, de um modo geral, as crianças pré-escolares mostraram desenvolver-se de forma progressiva, sendo que as meninas mostraram desempenhos sempre inferiores aos dos meninos.

O baixo desempenho das meninas deveria chamar a atenção dos professores, instrutores, educadores e os responsáveis por políticas públicas de saúde para a necessidade de programas de intervençóes com habilidades motoras, de forma a estimular a participação sistemática das meninas em habilidades motoras grossas, especialmente naquelas habilidades que tenham por meta o controle da força para propulsionar ou recepcionar objetos variados como bolas, petecas, tacos, entre outros.

\section{Abstract}

\section{Analysis of developmental levels in object control skills of preschoolers}

The intentional and controlled contact with objects is the goal of object control skills and it is expected that changes in such skills appear in a progressive sequence of developmental levels, with increasing chronological age. Sex is a factor to be further studied in this context. This study aimed to investigate the developmental levels (initial, intermediate and advanced) in object control skills of preschoolers, by age and sex. The sample consisted of 342 children aged 3, 4 and 5 years (187 boys). The TGMD-2 was used to evaluate the skills performance in strike, dribble, catch, kick, overhand throw, and underhand roll. Differences between groups and genders were analyzed using nonparametric tests; Chi-square test was used to identify the percentage 
of individuals in the developmental levels of each skill, according to age. The results showed that 5 year children were higher than 3 year in dribble, catch and total score of object control; in comparisons among children of 3 and 4 , and 4 and 5 years, the difference was in favor of the older children only in dribble and total score. The boys showed superior performance in five of the six skills and in total score. There was an association between the most advanced developmental levels and male gender for the total score (at 3 and 5 years), and specifically, to striking and underhand roll (4 years) and overhand throw (at 4 and 5 years) skills. In general, the performance of preschool children in object control skills improved with increasing age, indicating that motor development is cumulative and progressive.

\section{KeYwords: Child Development; Psychomotor Performance; Child; Motor Dexterity.}

\section{Referências}

1. Breslin G, Murphy M, Mckee D, Delaney B, Dempster M. The effect of teachers trained in a fundamental movement skills programme on children's self-perceptions and motor competence. Eur Phys Educ Rev. 2012;18:114-26.

2. Oliveira IS. O efeito de diferentes programas de intervenção no que se refere à instrução, sobre a competência motora e percepção de competência atlética de crianças [dissertação]. Recife: Programa Associado de Pós-graduação em Educação Física UPE/UFPB; 2014.

3. Logan SW, Robinson LE, Wilson AE, Lucas WA. Getting the fundamentals of movement: a meta-analysis of the effectiveness of motor skill interventions in children. Child Care Health Dev. 2012;38:305-15.

4. Clark JE. On the problem of motor skill development. J Phys Educ Recreat Dance. 2007;78:1-58.

5. Barnett LM, van Beurden E, Morgan PJ, Brooks LO, Beard JR. Does childhood motor skill proficiency predict adolescent fitness? Med Sci Sports Exer. 2008;40:2137-44.

6. Manoel EJ. Desenvolvimento motor: implicações para a educação física escolar I. Rev Paul Educ Fís. 1994;8:82-97.

7. Stodden DF, Goodway JD, Langendorfer SJ, Roberton MA, Rudisill ME, Garcia C, et al. A developmental perspective on the role of motor skill competence in physical activity: an emergent relationship. Quest. 2008;60:290-306.

8. Robinson LE, Stodden DF, Barnett LM, Lopes VP, Logan SW, Rodrigues LP, et al. Motor competence and its effect on positive developmental trajectories of health. Sports Med. 2015;45(9):1273-84.

9. Okely AD, Booth ML, Chey T. Relationships between body composition and fundamental movement skills among children and adolescents. Res Q Exerc Sport. 2004;75:238-47.

10. von Bertalanffy L. An outline of general system theory. Br J Philos Sci. 1950;I(2):134-65.

11. von Bertalanffy L. The history and status of general systems theory. Acad Manag J. 1972;15(4):407-26.

12. Haywood KM, Roberton MA, Getchell N. Advanced analysis of motor development. Champaign: Human Kinetics; 2012.

13. Diriwächter R, Valsiner J. Qualitative developmental research methods in their historical and epistemological contexts. Forum Qual Social Res [Internet]. 2006 [citado 2014 maio 31];7(1):1-21. Disponível em: http://bit.ly/2IKqp4s.

14. Ford DH, Lerner RM. Developmental systems theory: an integrative approach. Thousand Oaks: Sage Publications; 1992.

15. Booth ML, Okely AD, McLellan L, Phongsavan P, Macaskill P, Patterson J. Mastery of fundamental motor skills among New South Wales school students: prevalence and sociodemographic distribution. J Sci Med Sport. 1999;2:93-105.

16. Gallahue DL, Ozmun JC, Goodway JD. Compreendendo o desenvolvimento motor: bebês, crianças, adolescentes e adultos. $7^{\mathrm{a}}$ ed. Porto Alegre: Artmed; 2013.

17. Gallahue DL, Donnelly FC. Developmental physical education for all children. 4th ed. Champaign: Human Kinetics; 2003.

18. Williams HG, Pfeiffer KA, O’Neill JR, Dowda M, McIver KL, Brown WH, et al. Motor skill performance and physical activity in preschool children. Obesity. 2008;16:1421-6.

19. Hardy LL, King L, Farrell L, MacNiven R, Howlett S. Fundamental movement skills among Australian preschool children. J Sci Med Sport. 2010;13:503-8.

20. Silva SB, Vilela J, Guanis B, Toloka RE. Arremessar por cima do ombro e a distância percorrida pelo implemento. Rev Bras Educ Fís Esp [Internet]. 2009 [citado 2014 maio 31];23:309-18. Disponível em: http://bit.ly/2Xxk4Nh.

21. Haywood KM, Getchell N. Desenvolvimento motor ao longo da vida. 5a ed. Porto Alegre: Artmed; 2010.

22. Spessato BC, Gabbard C, Valentini N, Rudisill M. Gender differences in Brazilian children's fundamental movement skill performance. Early Child Dev Care. 2012;183(7):1-8.

Rev Bras Educ Fís Esporte, (São Paulo) 2018 Jan-Mar;32(1):109-19 • 117 
23. Afonso GH, Freitas DL, Carmo JM, Lefevre JA, Almeida MJ, Lopes VP, et al. Desempenho motor: um estudo normativo e criterial em crianças da Região Autónoma da Madeira, Portugal. Rev Port Ciênc Desporto. 2009;9:160-74.

24. Carvalhal M, Vasconcelos-Rapouso J. Diferenças entre gêneros nas habilidades: correr, saltar, lançar e chutar. Motricidade. 2007;3:44-56.

25. Villwock G, Valentini NC. Percepção de competência atlética, orientação motivacional e competência motora em crianças de escolas públicas: estudo desenvolvimentista e correlacional. Rev Bras Educ Fís Esp. 2007;21:245-57.

26. Azevedo JB. Nível de desempenho motor de crianças com 10 anos de idade em escolas publicas de Porto Alegre [trabalho de conclusão de curso]. Porto Alegre: Universidade do Rio Grande do Sul; 2009.

27. Castro MB. A influência do contexto nas habilidades motoras fundamentais de pré-escolares e escolares [dissertação]. Porto Alegre: Universidade Federal do Rio Grande do Sul; 2008.

28. Catenassi FZ, Marques I, Bastos CB, Basso L, Ronque VER, Gerage AM. Relação entre índice de massa corporal e habilidade motora grossa em crianças de quatro a seis anos. Rev Bras Med Esporte. 2007;13:227-30.

29. Hsin CT, Li MC, Tsai CC. The influence of young children's use of technology on their learning: a review. J Educ Techno Soc. 2014;17(4):85-99.

30. Yang SC, Lin SJ, Tsai CY. Effect of sex, age, and bmi on the development of locomotor skills and object control skills among preschool children. Percept Mot Skills. 2015;121(3):873-88.

31. Thomas JR, Nelson JK, Silverman SJ. Métodos de pesquisa em atividade física. 7a ed. Porto Alegre: Artmed; 2002.

32. Ulrich DA. Test of gross motor development-2. Austin: Prod-Ed; 2000.

33. Valentini NC. Validity and reliability of the TGMD-2 for Brazilian children. J Mot Behav. 2012;44:275-80.

34. Pansera SM, De Paula PR, Valentini NC. Educação física no ensino infantil: sua influência no desempenho das habilidades motoras fundamentais. Cinergis. 2008;9:24-32.

35. Silveira CRA, Gobbi LTB, Caetano MJD, Rossi ACS, Candido RP. Avaliação motora de pré-escolares: relaçóes entre idade motora e idade cronológica. Lect Educ Fís Desportes [Internet]. 2005 [citado 2013 maio 27];82. Disponível em: http://bit. ly/2TjwCZr.

36. Copetti F. Nível de maturação dos padrôes fundamentais de movimentos e o desempenho motor em pré-escolares. Cinergis. 2000;1:39-50.

37. Paim MCC. Desenvolvimento motor de crianças pré-escolares entre 5 e 6 anos. Lect Educ Fís Desportes [Internet]. 2003 [citado 2011 maio 27];8. Disponível em: http://bit.ly/2NzWhrk.

38. Cowan N. The development of working memory. In: Cowan N, Hulme C, organizadores. The development of memory in childhood. Hove: Psychology Press; 1997. p. 163-200.

39. Valentini NC. Percepçóes de competência e desenvolvimento motor de meninos e meninas: um estudo transversal. Movimento. 2002;8:51-62.

40. Smith LB, Thelen E. Development as a dynamic system. Trends Cogn Sci. 2003;7:343-48.

41. Brauner LM, Valentini NC. Análise do desempenho motor de crianças participantes de um programa de atividades físicas. Rev Educ Fís/UEM. 2009;20:205-16.

42. Valentini NC, Rudisill ME. An inclusive mastery climate intervention and the motor skill development of children with and without disabilities. Adapt Phys Act Q. 2004;21:330-47.

43. Goodway JD, Branta CF. Influence of a motor skill intervention on fundamental motor skill development on disadvantaged preschool children. Res Q Exerc Sport. 2003;74:36-47.

44. Malina RM, Bouchard C, Bar-Or O. Growth, maturation, and physical activity. 2a ed. Champaign: Human Kinetics; 2004.

45. Xavier CTS. A escola e o desenvolvimento motor em escolares [dissertação]. Belém: Universidade Federal do Pará; 2009.

46. Oliveira DS, Oliveira IS, Cattuzzo MT. A influência do gênero e idade no desempenho das habilidades locomotoras de crianças de primeira infância. Rev Bras Educ Fís Esporte 2013;27:649-57.

47. Newell KM. Constraints on the development of coordination. In: Wade MG, Whiting HTA, editors. Motor development in children: aspects of coordination and control. Dordrecht: Martinus Nijhoff; 1986. p. 341-60.

48. Lemos AG, Avigo E, Barela JA. Physical education in kindergarten promotes fundamental motor skill development. Adv Phys Educ. 2012;2:17-21.

49. Queiroz DR, Re AHN, Henrique RS, Moura MS, Cattuzzo MT. Participation in sports practice and motor competence in preschoolers. Motriz. 2014;20(1):26-32.

50. Oliveira IS, Oliveira DS, Cattuzzo MT. The effect of different instructions in a general motor competence and perceived competence of children. J Phys Educ Sport Manag. 2016;3(1):108-126. 
51. Cattuzzo MT, Henrique RS, Ré AH, de Oliveira IS, Melo BM, de Sousa Moura M, et al. Motor competence and health related physical fitness in youth: a systematic review. J Sci Med Sport. 2016;19(2):123-9.

52. Cattuzzo MT, Beltrão NB, Campos CMC, Guerra ERF. A multicausalidade desenvolvimental: uma abordagem sistêmica da proficiência motora e da prática da atividade física. In: Cattuzzo MT, Caminha IO, organizadoras. Fazer e pensar ciência em educação física: livro 1. João Pessoa: UFPB; 2012. cap. 3.

\begin{tabular}{r|l} 
ENDEREÇo & \\
Maria Teresa Cattuzzo & Recebido para publicação: 03/07/2014 \\
Escola Superior de Educação Física, & 1a. Revisão: 04/05/2016 \\
Universidade de Pernambuco & 2a. Revisão: 11/11/2016 \\
Arnobio Marques, 310 - Santo Amaro & 3a. Revisão: 10/03/2017 \\
30 - Recife - Pernambuco - BRASIL & Aceito: 17/04/2017 \\
e-mail: mtcattuzzo@hotmail.com &
\end{tabular}


